\section{Moving towards tissue-engineered disc replacement}

Tissue-engineered disc replacements are a potential alternative option to fusion surgery for the treatment of severe intervertebral disc (IVD) degeneration. In a new study, researchers report on the successful long-term integration and mechanical function of engineered discs in vivo, including in a large animal model, moving this approach a step towards translational feasibility.

In previous short-term studies, the researchers had tested various iterations of a disc-like angle ply structure (DAPS) in a rat disc replacement model. These structures have distinct components that mimic the structure of the native disc. "Our latest iterations of the DAPS includes an 'endplate' that promotes integration between the native vertebral bodies and our engineered implant," explains Robert Mauck, corresponding author of the new study.
The eDAPS sized for the rat caudal spine maintained its structure and composition 20 weeks after in vivo implantation in the rat tail.

Data from various assessments including second harmonic generation (SHG) imaging suggested that the eDAPS functionally integrated with the native discs at the endplate interfaces. The mechanical properties of the eDAPS stayed the same or improved after 20 weeks, with the tensile and compressive near-native values.

The researchers also tested eDAPS sized for the human cervical disc space in a goat cervical disc replacement model. The findings paralleled those of the rat model, with the eDAPS compositionally and functionally maturing after 8 weeks in vivo. to humans is a real and distinct mechanical properties reaching

"Given these findings, translation

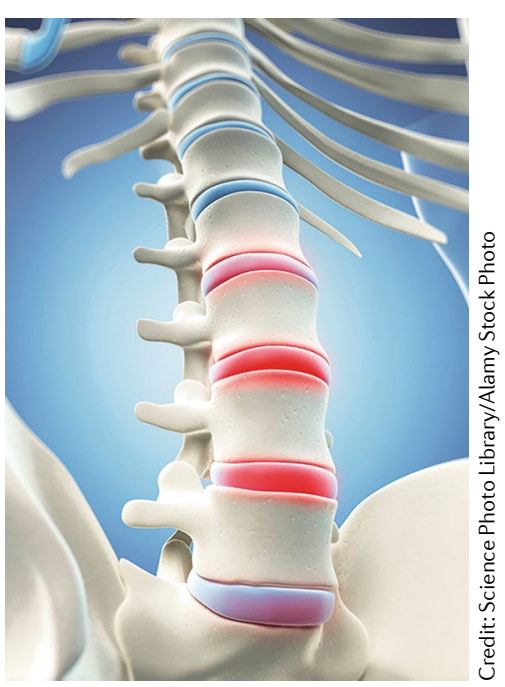

possibility," explains Mauck.

"There is of course quite a lot to do in our goat model, such as longer term evaluation, aggressive remobilization and implantation into a diseased (rather than an acute defect) environment, before we can move on to human clinical trials."

Jessica McHugh improved after 20 weeks

5

SYSTEMIC LUPUS ERYTHEMATOSUS

\section{Dietary modulation of the microbiome as therapy}

Microbial fermentation of starches in the intestine produces shortchain fatty acids (SCFAs) that can modulate host immunity and potentially reduce susceptibility to inflammatory diseases. New work published in Cell Host \& Microbe now shows that intestinal Lactobacillus reuteri contributes to disease in mouse models of systemic lupus erythematosus (SLE) and this population can be inhibited by feeding mice a diet rich in starches that are resistant to intestinal digestion and thereby available for microbial fermentation into SCFAs.

"We used transgenic mice that overexpress the innate RNA-sensing receptor TLR7 because it is a very good model to study the innate immune lupus-like disease

5

arm of the pathogenesis of SLE," explains Martin Kriegel, corresponding author of the new article. "These mice develop all the hallmarks of human SLE via the increased signalling of TLR7, which activates plasmacytoid dendritic cells to secrete IFNa, a signature cytokine in SLE."

A resistant starch diet suppressed the growth of L. reuteri in these lupussealed the gut barrier," explains Kriegel. "A fluorescent dye fed to the mice was not able to leak into the systemic circulation and fewer live bacteria translocated to tissues."

The researchers also showed that seeding the intestines of mice with L. reuteri exacerbated lupus-like disease and increased permeability of the intestinal barrier and translocation of bacteria into the lymph nodes, spleen and liver.

Kriegel now wants to study more of the underlying biology of the protection afforded by diet and how L. reuteri is pathogenic. "We would like to identify metabolites that are secreted by L. reuteri that might activate plasmacytoid dendritic cells," he says, for example. prone mice. "In addition, the diet

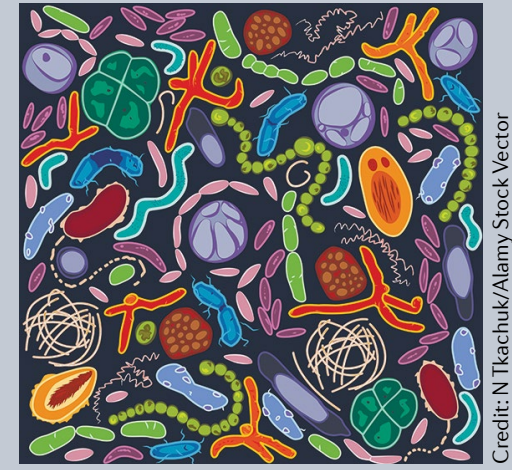

The researchers also provide some human relevance to the study by showing that Lactobacillus species are enriched in faecal samples taken from a subset of patients with SLE. However, the main question Kriegel would like to address is whether or not a dietary intervention involving resistant starches would be therapeutically beneficial for patients with SLE.

Nicholas J. Bernard
ORIGINAL ARTICLE Zegarra-Ruiz, D. F. et al. A diet-sensitive commensal Lactobacillus strain mediates TLR7-dependent systemic autoimmunity. Cell Host Microbe S1931-3128, 30591-30592 (2018) 